\title{
THE REDSHIFT EVOLUTION OF CIV IN GALAXY HALOS
}

\author{
E. A. STENGLER LARREA \\ Royal Greenwich Observatory \\ Madingley Road, Cambridge CB3 OEZ, UK
}

Improved and reliable profile fitting codes have made it recently possible to calculate reliable column densities of gas producing QSO absorption lines. Even using intermediate to low resolution spectra, the obtained column densities are very accurate, as has been extensively proved by Jenkins (1986). We applied this technique to the 131 CIV absorption systems in the survey by Sargent Boksenberg and Steidel (1988) and Steidel (1990). Since several lines were saturated, many CIV column densities, $N(\mathrm{CrV})$, are only lower limits, and a survival analysis (Avni et al. 1980) is required to obtain the underlying redshift distribution of CIV column densities, which we present in Figure 1. Together with the number density distribution of strong $(\log N(\mathrm{CIV}) \geq 14.2)$ and weak systems shown in Figure 2, we have found that $\mathrm{I}$. Both strong and weak systems become generally weaker below $z \sim 2$, and II. Weak systems become less numerous below $z \sim 2$, whereas the stronger systems grow steadily in number density down the lowest redshifts sampled. For details of the analysis, and on how this fits into a model for the CIV absorption systems see Stengler-Larrea et al. (1995, in preparation).

References Avni, Y., Soltan A., Tananbaum H., and Zamorani, G. 1980, Ap.J. 238, 800 . Jenkins, E.B. 1986, Ap.J. 304, 739. Sargent, W.L.W., Boksenberg, A., and Steidel, C.C. 1988, Ap.J.S. 68, 539. Steidel, C.C. 1990, Ap.J.S. 72, 1.

Figure 1 (left) Redshift distribution of the CIV column densities obtained applying a survival analysis to the results of profile fitting $131 \mathrm{Crv}$ systems.

Figure 2 (right) Redshift distribution of the number density of the CIV systems in our sample, for the weak (solid) and strong (dashed) subsamples.
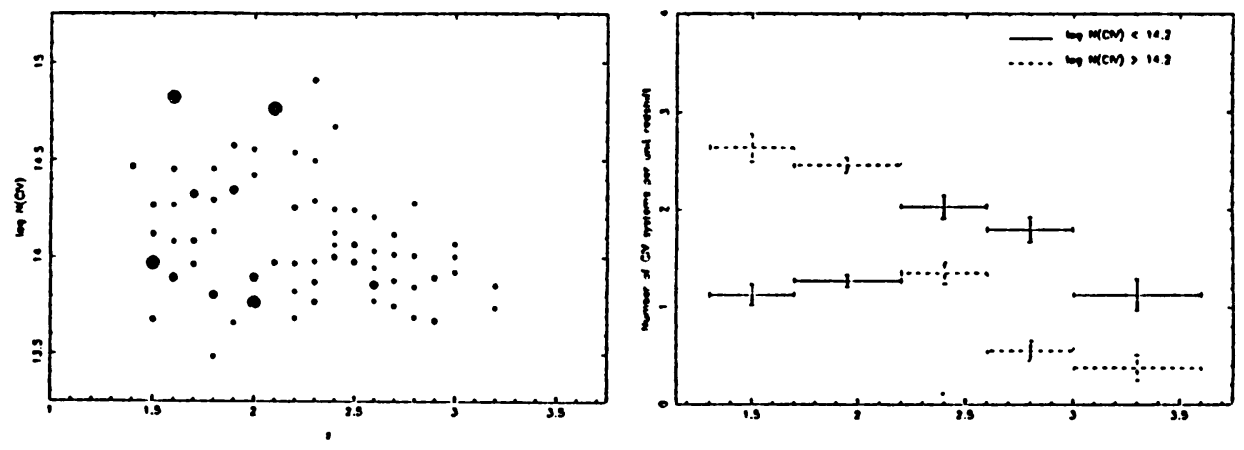